\title{
PARTICIPACIÓN POLÍTICA DE LAS MUJERES. ASPECTOS DE CONFLUENCIA ENTRE LA ÉTICA Y LOS DERECHOS HUMANOS
}

\begin{abstract}
Tatiana Rein Venegas ${ }^{1}$
Resumen: Este artículo explora los vínculos entre ética global y derechos humanos, con un foco particular en la participación política de las mujeres, como una forma de indagar en las posibles ampliaciones al campo de la ética global. Para ello, se determinan los alcances de los conceptos de ética y de ética global, así como de derechos humanos, y se examinan los vínculos entre ellos, particularmente la incorporación de consideraciones éticas por los derechos humanos y la fuerza que éstos les otorgan. Finalmente, el artículo se centra en los derechos de las mujeres y, específicamente, en la participación política; se analizan diversas alternativas, que van de las tradicionales de participación parlamentaria a formas como el establecimiento de agencias gubernamentales para mejorar la condición de las mujeres, y a formas de intervención a través de movimientos de mujeres. Se concluye apelando a la necesidad de que, tanto desde la ética como de los derechos humanos, se evalúen fórmulas para lograr la implementación de estos derechos que, en parte, pueden derivar de la incorporación más permanente de los movimientos de mujeres en la toma de decisión de políticas públicas.
\end{abstract}

Palabras clave: participación política de las mujeres, derechos humanos, ética global, movimientos de mujeres

\section{Political participation of women. Some confluences between ethics and human rights}

\begin{abstract}
This article explores the links between global ethics and human rights, with a particular focus on women's political participation, as a way of inquiring into possible ramifications within the field of global ethics. To this end, it determines the scope of the concepts of ethics and global ethics as well as that of human rights. It analyses the links between them, particularly the incorporation of ethical considerations on the part of human rights and the validity that the latter assign to them. Finally, the article concentrates onto women's rights and more specifically on their political participation. It analyses the diverse alternatives of participation, which cover a range that spans from the traditional parliamentary participation up to forms like the establishment of governmental agendas aimed at improving the condition of women and the participation through women's movements. It is concluded that, both from an ethics as well as human-rights point of view, it is necessary an evaluation of ways for achieving the implementation of these rights. These can derive from a more permanent incorporation of women's movements in the decision making process of public policies.
\end{abstract}

Key words: women's political participation, human rights, global ethics, women's movements

Participaçáo política das mulheres. Aspectos de confluência entre a ética e os direitos humanos

Resumo: Este artigo explora os vínculos entre ética global e direitos humanos, com um foco particular na participação política das mulheres, como uma forma de indagar as possíveis ampliaçôes ao campo da ética global. Para isso, são determinados os alcances dos conceitos de ética e de ética global, assim como de direitos humanos. São analisados os vínculos entre eles, particularmente a incorporaçáo de consideraçôes éticas pelos direitos humanos e a força que estes lhes outorgam. Finalmente, o artigo se centra nos direitos das mulheres e, especificamente, na participação política. São analisadas diversas alternativas, que vão desde as tradicionais de participação parlamentar a formas como o estabelecimento de agências governamentais para melhorar a condição das mulheres, e as formas de intervenção através de movimentos de mulheres. Conclui-se apelando para a necessidade de que, tanto a partir da ética como dos direitos humanos, se avaliem fórmulas para lograr a implementação destes direitos, que em parte podem derivar da incorporação mais permanente dos movimentos de mulheres na tomada de decisão de políticas públicas.

Palavras-chave: participação política das mulheres, direitos humanos, ética global, movimentos de mulheres

\footnotetext{
${ }^{1}$ Instituto de Estudios Internacionales, Universidad de Chile, Chile Correspondencia: trein@uchile.cl
} 


\section{Introducción}

El presente artículo surge en el contexto de un seminario en Ética Global organizado por el Instituto de Estudios Internacionales y el Centro Interdisciplinario de Estudios en Bioética de la Universidad de Chile, denominado "problemas actuales de la ética global" 2 , que tuvo por objeto indagar la posible ampliación del campo temático de la ética global. Con dicho objetivo en mente, este artículo explora los vínculos entre ética global y derechos humanos, concretamente un derecho específico de un sector de la población: la participación política de las mujeres.

Este artículo cuenta con dos secciones preliminares dedicadas a determinar qué se entiende por ética y por el más actual concepto de ética global, con el fin de establecer los alcances de ambos términos y los usos que de ellos se ha hecho. Así, se establecen las conexiones entre el concepto de ética y el de responsabilidad, así como de respeto por los derechos de los otros. Se establece la extensión del concepto de ética global y la necesidad de buscar soluciones a problemas que van más allá de las fronteras nacionales. Por último, se deja establecida la relación de estos conceptos y el de poder, que realmente otorga la posibilidad de tomar opciones de respeto por los derechos de los otros y de buscar soluciones conjuntas. Una tercera sección explica conceptos generales sobre derechos humanos y las obligaciones que establecen para los Estados.

Una cuarta sección indaga en la relación entre ética y derechos humanos, estableciendo un reconocimiento a los vínculos entre estos conceptos. Así, se señala que los derechos humanos incorporan consideraciones éticas y les dan fuerza. Posteriormente se aborda la crítica, tanto a las concepciones éticas como de derechos humanos, desde las perspectivas de derechos de las mujeres, las que apuntan principalmente a que, pese al reconocimiento a estos derechos, falta mucho para su implementación. Finalmente, se abordan las diversas alternativas de participación política que tienen las mujeres, en términos de política representativa en los parlamentos y a través de entidades gubernamentales creadas para mejorar su

${ }^{2}$ Seminario que tuvo lugar el 16 y 17 de mayo de 2013, en la sede del Instituto de Estudios Internacionales de la Universidad de Chile. condición, todas las cuales, si bien han logrado importantes avances, requieren de grandes esfuerzos para que las mujeres se encuentren en condiciones de igualdad en la toma de decisiones políticas. Por último, me refiero a los movimientos de mujeres, a su rol en la incorporación de asuntos de las mujeres en la agenda política y la necesidad de buscar maneras de incorporarlos a través de fórmulas más permanentes en la determinación de la agenda política.

\section{Moral y ética: esbozando conceptos}

El concepto de ética se relaciona estrechamente con el concepto de moral. Este último es un término que se usa en una variedad de sentidos, uno de los cuales se refiere a las normas de comportamiento que establecen lo correcto y lo incorrecto en la conducta de individuos en una sociedad específica(1). En esa misma dirección, puede ser entendido como la reflexión en torno a cuáles deben ser los estándares de conducta, así como los deberes de los miembros de una $\operatorname{sociedad}(1)$. La ética, por su parte, se refiere a la reflexión sobre problemas morales y juicios de valor(2). Así entendidos, moral y ética son conceptos estrechamente vinculados que establecen pautas de conducta a los miembros de una sociedad, de acuerdo con criterios valóricos de lo bueno y lo malo, lo correcto y lo incorrecto. Se trata de preferencia de unos valores sobre otros, entre los cuales el valor ético per se es la justicia, que busca determinar lo bueno y correcto y, en tal sentido, también surgen los derechos(3), sobre los cuales se hará referencia más adelante en esta misma sección

En el ámbito académico, sin embargo, el término ética no ha sido extensamente utilizado hasta ahora, fuera del campo de la filosofía. Sus principales usos han estado relacionados al ámbito de la medicina principalmente y al de la metodología de la investigación, en segundo plano(4). Respecto del primero, se utiliza en la discusión del actuar de los investigadores, de acuerdo con estos criterios morales fundamentales. Así, por ejemplo, se exige el respeto por la vida, en todas sus formas (2,5). Además se establece una especie de "imperativo para la salvación de la especie humana mediante la solidaridad con la biósfera" (5). En estos sentidos, así como en otra variedad de aspectos médicos, el concepto de ética se ha utilizado más como "bioética" (4). 
Por su parte, en el ámbito de la metodología de la investigación, algunos ejemplos del uso de consideraciones éticas dicen relación con aquella investigación o tratamiento que involucra seres humanos, verbi gratia, la autorización que debe dar el paciente, la cual debe ser informada. Si bien hasta recientemente este uso no estaba del todo extendido en las ciencias sociales, hoy en día ya no es posible, por ejemplo, realizar entrevistas personales para una investigación sin tener la aprobación de un comité de ética. Éste autoriza la realización de las entrevistas solo en el caso que no se esté poniendo de ningún modo en riesgo a individuos(6). También hay resguardos adicionales que tomar, por ejemplo, mantener el anonimato de los entrevistados o cambiar la forma de formular una pregunta cuando trata de asuntos sensibles(6). Un típico caso en que este último escenario se podría dar es al desarrollar una investigación sobre violencia sexual contra mujeres en tiempos de conflicto armado, en la cual las entrevistadas son las propias víctimas.

Cuando se piensa en los ejemplos señalados en los párrafos anteriores se pueden ver las conexiones entre el concepto de ética y los conceptos de derecho y de responsabilidad. En este sentido, la ética ha sido concebida como la organización de derechos y deberes(3), e implica considerar la existencia de los derechos de otros, lo que a su vez se traduce en la responsabilidad de respetar esos derechos. Como consecuencia, si cada persona tiene la obligación de respetar los derechos de los otros, los propios derechos se encontrarán, a su vez, asegurados(3). Este intercambio de derechos y deberes conlleva un sentido de responsabilidad, lo que podría llamarse "sistema de interacciones éticas" $(2,3)$.

En consecuencia, la ética moderna conlleva la idea de responsabilidad, en el sentido que las personas deben respetar los derechos de los otros, pero también en el sentido que los individuos tienen el poder de decidir libre y autónomamente el tipo de persona que quieren ser(3). Derivado de esto y dado que la decisión final sobre respetar o no los derechos de los otros depende de la voluntad de los individuos, existe el riesgo que éstos actúen sobre la base de su interés personal por sobre el colectivo, vulnerando los derechos de sus semejantes, lo cual se ve agravado porque dependemos los unos de los otros y, entonces, las consecuencias de las decisiones de unos sobre el respeto o no de los derechos de los otros sin duda nos afectarán(3).

Las dimensiones de ética abordadas hasta ahora brindan contenido al concepto de ética global, un concepto que está empezando a ser utilizado cada vez de manera más amplia y que va más allá de los aspectos mencionados, para surgir como concepto independiente, con contenido propio. En lo que sigue me detendré en la determinación de dicho contenido, para luego vincularlo a los conceptos de derechos humanos y derechos de las mujeres, en particular a la participación política de éstas, como un derecho humano, como un requisito de la democracia y como un imperativo ético.

\section{Ética global}

Ética global es un concepto vinculado con una serie de disciplinas de las ciencias sociales, como las relaciones internacionales, los derechos humanos y las teorías feministas(3,7-9). Cuando se habla de ética global se alude, más que a un término geográfico(8), a un sentido que trasciende a una comunidad específica, también hace referencia a la necesidad de integrar y contrastar discursos diversos(8). En este sentido, se opondría a imposiciones de ideas desde ciertos grupos sobre otros y vendría a enfrentar ciertas prácticas que se han dado en el ámbito de las relaciones internacionales en general y de los derechos humanos en particular.

El concepto de ética global, desde esta perspectiva, ayuda a identificar una serie de problemas que cruzan las fronteras nacionales o que son compartidos por grupos de personas, más allá de su ubicación geográfica, inter alia, contaminación ambiental, cambio climático, crimen internacional, conflicto militar, terrorismo, así como pobreza, hambruna, malnutrición y discriminación(7). Estos problemas solo pueden ser resueltos o mitigados con un esfuerzo coordinado de la comunidad mundial(7).

El concepto de ética global ha crecido y se ha expandido en la última década(7). Se relaciona con la necesidad de identificar valores morales y normas para avanzar en el bienestar de los seres humanos y sus relaciones con otras entidades del 
planeta(7). Contiene una visión holística moral de paz, relaciones armoniosas, equidad y justicia, y desarrollo sustentable(7). Sin embargo, existe una visión de este concepto que lo relaciona con el concepto de globalización en un sentido económico, economías neoliberales, y hegemonía económica occidental. La relación con la globalización tiene algunas implicancias específicas: en primer lugar, no se puede discutir de ética sin aludir al tema del poder; en segundo término, la globalización ha implicado una imposición de una esquema local -y más concretamente el occidental — a la población global(8).

El Institute for Global Ethics ${ }^{3}$ es una organización que explota la base de valores comúnmente compartidos para promover una acción ética. Ellos han definido ciertos parámetros de la ética global. Entre ellos, los requerimientos mínimos de buena vida para todas las personas, que incluyen educación, seguridad física y niveles apropiados de servicios de salud. Por otra parte sostienen que, en el plano nacional, se debe asegurar respeto por la vida, libertad, igualdad, justicia, acceso igualitario a oportunidades para el desarrollo personal, y participación política en todos los niveles de toma de decisión para asegurar el bien común(7). Este punto, así como el señalado sobre la necesidad de discutir sobre el tema del poder al hablar de un mundo global, son sin duda aspectos cruciales al abordar el tema de la participación política de las mujeres, a la que me referiré más adelante en este artículo. Finalmente, entre los parámetros de ética global y considerando un ámbito internacional, el instituto recomienda fomentar un ambiente que tenga como objetivo la equidad económica entre naciones, el acceso a bienes comunes en condiciones de igualdad, así como la cooperación en el combate de problemas compartidos(7).

De esta manera, hoy se han ampliado las posibilidades de tomar opciones, y que éstas sean opciones éticas. El poder es un concepto esencial, que otorga la posibilidad de tomar opciones informadas, y de que las decisiones adoptadas tengan algún nivel de influencia(3). De este modo, saber quiénes acceden a posiciones de poder y pueden participar en la toma de decisiones, tanto individual como globalmente, se vuelve crucial.

\footnotetext{
${ }^{3}$ Ver http://www.globalethics.org/ (último acceso 03/09/13).
}

\section{Derechos humanos}

Las dos secciones previas abordaron temas conceptuales relacionados con lo que se entiende por ética y más especialmente lo que hoy en día se entiende por ética global. Esta sección vinculará estas aproximaciones conceptuales a la temática de los derechos humanos. En este sentido, conviene recordar que es solo con posterioridad a las guerras del siglo XX que se reconoció el sistema de derechos humanos como un mínimo que los Estados debían respetar. Posteriormente, luego de la Guerra Fría, la democratización del sistema internacional adquirió especial importancia y favoreció una perspectiva más ética de solución de los problemas internacionales. Así, los derechos humanos, la justicia penal internacional, los asuntos medioambientales, la paz, el desarrollo, la democracia, por enumerar algunos, han pasado a ser asuntos centrales del discurso público. Sin embargo, aún en estos días estamos lejos de encontrar una nueva agenda que reemplace las nociones tradicionales de poder y seguridad, nociones fuertemente influenciadas por teorías realistas de las relaciones internacionales, que se oponen a la incorporación de aspectos éticos $(3,10)$.

El derecho tiene cabida en esta discusión ya que, teniendo presente que los individuos actúan conforme a intereses personales, el derecho es necesario para regular las relaciones entre ellos y el Estado, así como entre las personas y otras instituciones. De este modo, viene a asegurar ese mínimo de ética necesario para vivir de manera relativamente armoniosa. Los derechos humanos, por su parte, se aseguran a todas las personas simplemente por ser personas(11). Son iguales para todos y no se puede renunciar a ellos, como tampoco se puede renunciar a la calidad de persona. Así, la fuente de los derechos humanos está en la naturaleza inherente de la persona humana(11).

Como los derechos humanos tienen una dimensión ética, algunos argumentaron que eran una parte inapropiada o que, al menos, no correspondían al ámbito de la política internacional(10). Sin embargo, en las últimas décadas los derechos humanos se han aceptado como una parte del estudio y de la práctica de la política internacional, aun cuando siguen siendo un interés secundario para muchos Estados y organizaciones internacionales y regionales $(10,12)$. 
Los derechos humanos han tenido un gran desarrollo. En efecto, desde 1948, fecha de la Declaración Universal de Derechos Humanos, los Estados han ratificado una extensa lista de tratados que velan tanto por los derechos humanos en general, como por derechos específicos que requieren de protección especial, como los de grupos o sectores de la población ${ }^{4}$. Los Estados han ratificado estos tratados tanto en el plano global, en el seno de Naciones Unidas, como en el marco de los sistemas regionales de protección de los derechos humanos $(13)^{5}$.

La mayor parte de los estados ha ratificado o adhiere a los tratados más importantes de derechos humanos ${ }^{6}$. La Convención sobre la eliminación de todas las formas de discriminación contra la mujer (CEDAW), por ejemplo, cuenta con 187 ratificaciones a la fecha ${ }^{7}$, aun cuando también tiene reservas $\tan$ importantes que incluso atentan contra su propio espíritu. Por otra parte, el lenguaje de los derechos humanos ha estado en el centro de la retórica de la diplomacia bilateral y multilateral de la política contemporánea y los llamados que legítimamente hacen los Estados.

Los derechos humanos imponen a los Estados la obligación de respetar, proteger e implementar los derechos humanos(14). Los Estados deben, por una parte, abstenerse de violar este tipo de derechos y proteger a las personas de que otros violen sus derechos; por otra, deben establecer las condiciones para que las personas los puedan disfrutar. De esta manera, no se trata solo de una obligación pasiva, sino de adoptar políticas, leyes

\footnotetext{
${ }^{4}$ Para una lista de los tratados internacionales de derechos humanos ver: http://www2.ohchr.org/spanish/law/index.htm (último acceso el 5 de septiembre de 2013).

${ }^{5}$ Los sistemas regionales de protección de los derechos humanos son el Sistema Interamericano, el Europeo y el Africano de Derechos Humanos, y se enmarcan en el seno de organizaciones regionales, como la Organización de Estados Americanos, la Unión Europea o la Organización para la Unidad Africana(13).

${ }^{6}$ A la fecha, 167 Estados ha ratificado el Pacto Internacional de Derechos Civiles y Políticos, 160 ha ratificado el Pacto Internacional de Derechos Económicos, Sociales y Culturales, por nombrar sólo los tratados que forman parte de la llamada Carta Internacional de Derechos Humanos. Para mayor información, ver http://treaties. un.org/Pages/Treaties.aspx?id=4\&subid=A\&lang=en (último acceso el 5 de Septiembre de 2013).

${ }^{7}$ Ver http://treaties.un.org/Pages/ViewDetails. aspx?src=TREATY\&mtdsg_no=IV-8\&chapter=4\&lang=en (último acceso el 4 de septiembre de 2013)
}

u otras condiciones en general para garantizar que los individuos gocen efectivamente de sus derechos(14).

\section{Relación entre derechos humanos y ética}

Las teorías morales, que indican que se debe hacer lo correcto, dicen poco sobre derechos. Si bien ambos ámbitos están estrechamente relacionados, los derechos humanos no han sido especialmente tenidos en cuenta por las teorías morales(10). Los derechos humanos, por su parte, sí reconocen presupuestos de la ética y los han desarrollado, les han dado fuerza, en el sentido que los Estados se han comprometido a respetar estos supuestos y han creado instituciones para ello(10). De todos modos, los derechos humanos son violados constantemente y, por lo mismo, además de las instituciones establecidas para velar por su cumplimiento $^{8}$, hay una serie de mecanismos que se dirigen en el mismo sentido, tales como actividades diplomáticas encaminadas a persuadir a los Estados de la necesidad de actuar protegiendo los derechos humanos y actividades realizadas por la sociedad civil organizada orientadas a dejar en evidencia a los Estados sobre las violaciones de derechos que cometen en sus territorios $(18,19)$, todos ámbitos en los que el concepto de poder pasa a ser fundamental, lo que se refleja en que países más poderosos son menos susceptibles de presión para que modifiquen sus actividades violatorias de derechos $(20,10)$.

Hay dimensiones políticas, morales y legales en el desarrollo de los derechos humanos. Todas las sociedades apelan a valores como la equidad, compasión, respeto por los otros(10); sin embargo, pocas se refieren, al hablar de los otros, a todos los otros, a todos los miembros de la especie humana. Esta es la postura adoptada por la sociedad internacional contemporánea, sin perjuicio de lo cual falta aun bastante para que esto sea una realidad. Son necesarias algunas consideraciones adicionales para que los derechos humanos se acerquen más a los presupuestos de la ética glo-

\footnotetext{
${ }^{8}$ Tanto el sistema universal de protección de los derechos humanos como los sistemas regionales, han creado órganos que velan por el cumplimiento de los derechos establecidos en los tratados de derechos humanos. Algunos de estos órganos han contemplado, además de mecanismos de información, la posibilidad de que individuos o grupos de individuos presenten quejas contra Estados por la violación de sus derechos(15-17).
} 
bal y se refieren a asegurar que se respeten los de ciertos sectores de la población y grupos minoritarios. Normalmente esas consideraciones y el respeto de los derechos de estos sectores son el resultado de movimientos sociales, en un proceso que va desde la base hacia las cúpulas $(20,10)$, y estos proyectos no solo se refieren a los derechos civiles y políticos, sino que incluyen necesidades socioeconómicas y de desarrollo, e incluso aspectos medioambientales.

\section{Derechos humanos de las mujeres: crítica a la ética y a los derechos humanos}

Los derechos humanos, de acuerdo con su definición y al tener como base los principios de igualdad y no discriminación, debieran ser iguales para todas las personas, sin distinciones de ninguna especie(11). Así concebidos, los Estados están supuestamente obligados a garantizar la debida protección de todas las personas; sin embargo, los derechos humanos surgen en un cierto contexto $y$, al no mencionar específicamente a las mujeres, éstas no necesariamente han accedido a estos derechos en un plano de igualdad, por lo cual han debido exigir se reconozca que son acreedoras de los mismos derechos(21). Además han debido exigir el reconocimiento de ciertas vulneraciones de derechos a las que están particularmente ex$\operatorname{puestas}(21)$.

En este contexto, los movimientos de mujeres han realizado una labor extraordinaria al visibilizar y exigir el reconocimiento de derechos: por ejemplo el derecho a voto, a vivir una vida libre de violencia, igualdad en el acceso a derechos civiles y políticos, incluso derecho al acceso a la educación(21). Sin embargo, tanto los marcos teóricos como las creencias y las instituciones siguen marginando a las mujeres a un rol secundario, siguen sin protegerlas de violaciones a derechos que particularmente las afectan, como la violencia doméstica, el tráfico de mujeres y la ablación femenina(21). Tampoco les garantizan el goce de derechos consagrados, como la participación política efectiva.

Los movimientos de mujeres han luchado continuamente por lograr reconocimiento y protección efectiva de sus derechos de las mujeres, pero esto implica un arduo trabajo que implica cambiar mentalidades e instituciones(21). Para ello, una estrategia es lograr instituciones que velen por sus derechos, otra es acceder a puestos de poder y otra es vincularse con las personas directa o indirectamente para cambiar mentalidades $(22,23)$. Sin embargo, todas estas estrategias implican un mayor acceso a tomas de decisión, una participación en la definición de la agenda pública, y la influencia en la formación de preferencias de las personas, todos aspectos que forman parte definitoria de lo que es poder(24)y, por lo tanto, implica enfrentar a quienes actualmente detentan ese poder.

\section{Participación política de las mujeres}

Los derechos de participación política son algunos de los que han sido expresamente reconocidos a las mujeres. La convención sobre la eliminación de todas las formas de discriminación contra la mujer (CEDAW) ${ }^{9}$, en su artículo 7 , exige a los Estados tomar medidas apropiadas para que las mujeres accedan, en condiciones de igualdad, al derecho a votar y ser elegibles, participar en la formulación y ejecución de políticas públicas, ocupar cargos públicos, así como participar en organizaciones no gubernamentales que se ocupen de la vida pública y política. Sin embargo, en la práctica estos derechos están lejos de ser una realidad. El gráfico 1 refleja el porcentaje de mujeres legisladoras en los parlamentos en términos mundiales, en tanto el gráfico 2 muestra porcentajes de mujeres legisladoras en parlamentos latinoamericanos.

Gráfico 1. Mujeres legisladoras en los parlamentos del mundo

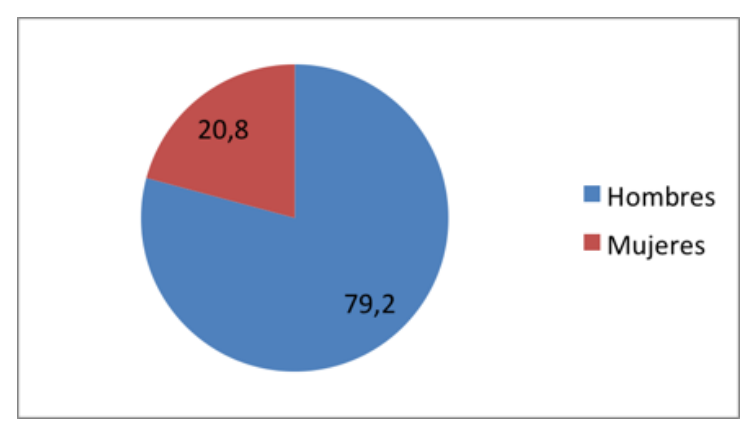

Fuente: Elaboración de la autora de acuerdo a datos de Interparliamentary Union, tomando en cuenta los parlamentos del mundo al 1 de abril de 2013 (www.ipu.org).

${ }^{9}$ Convención aprobada por la Asamblea General de Naciones Unidas en 1979 y que entró en vigor en 1981. 
Gráfico 2. Mujeres legisladoras en parlamentos latinoamericanos.

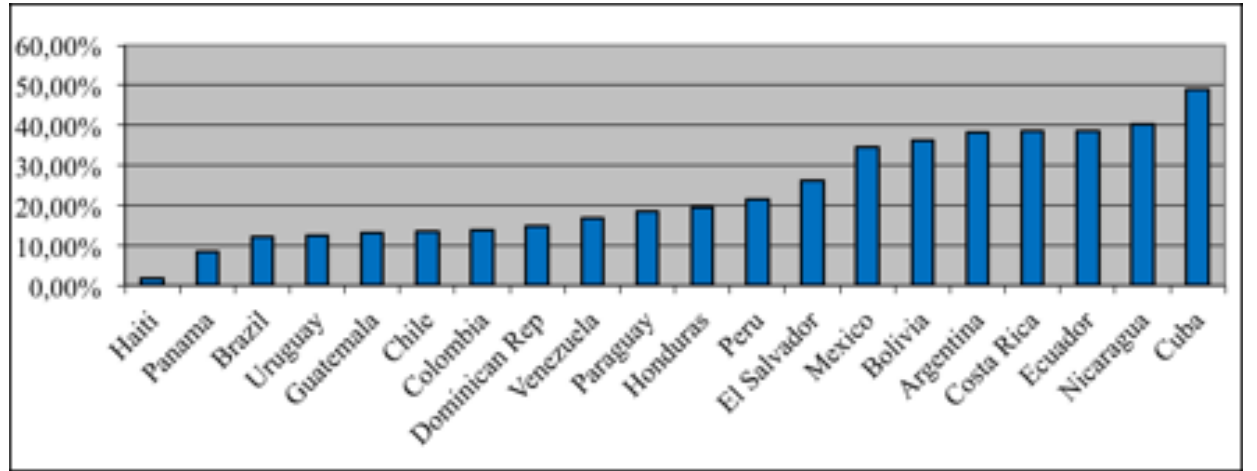

Fuente: Elaboración de la autora de acuerdo a datos de Inter-parliamentary Union, tomando en cuenta los parlamentos del mundo al 1 de julio de 2013 (www.ipu.org).

Las cifras muestran que aún falta mucho para que las mujeres realmente ocupen cargos públicos en condición de igualdad, aun cuando se ha avanzado en dicha dirección. Los casos de parlamentos con mayores porcentajes de mujeres se deben a que se han adoptado leyes de cuotas $\mathrm{u}$ otras medidas específicas para avanzar en una mayor igualdad $(25)^{10}$. Sin cuotas u otras medidas de tipo electoral es muy difícil que las mujeres puedan llegar a ocupar posiciones de poder $(25,27)$.

Razones de diversa índole justifican que aumente el número de mujeres en puestos de poder, tales como: el modelo que representan para otras mujeres; razones más éticas vinculadas a justicia entre los sexos; la representación de intereses que sin su presencia no serán incluidos o estarán subrepresentados; aquellas que sugieren que las mujeres se relacionan con la política de manera diferente a los hombres y que, por lo tanto, mejorarán la calidad de la vida política, y aquellas que sostienen que la falta de representación de las mujeres perjudica la calidad de la democracia $(28,25)$. La mayoría de estas razones se relacionan con los principios éticos a que se ha hecho referencia y pueden ser abordadas con medidas legales, como reformas a los sistemas electorales o la incorporación de medidas especiales, como las cuotas.

La participación política de las mujeres en puestos de representación es muy necesaria, por las razones de democracia, de representación de in-

\footnotetext{
${ }^{10}$ Sin perjuicio de lo cual, hay casos en los cuales los porcentajes de parlamentarias han aumentado sin existencia de cuotas o en los que, pese a la existencia de cuotas, no hay un incremento del porcentaje de mujeres en el parlamento (www.ipu.org) $(25,26)$.
}

tereses, entre otras de las ya señaladas. Sin embargo, esta no es la única forma en que las mujeres pueden acceder a posiciones de toma de decisión o de influencia de decisión ${ }^{11}$. Otra alternativa es lograr el establecimiento y luego la dirección de estructuras gubernamentales con el propósito de mejorar el estatus social de las mujeres, llamadas women's policy agencies (29). Women's policy agencies es un concepto desarrollado por una perspectiva llamada state feminism y que se refiere a estructuras permanentes o ad hoc, nacionales o regionales, departamentos u oficinas dentro del aparato estatal o ministerios, establecidas por el gobierno con el propósito de mejorar el estatus social de la mujer $(29,30)$. Existe una gran variedad en el poder de estas agencias de influir la formulación de políticas, así como en el nivel de organización que alcanzan(29). En el ámbito nacional, el ejemplo de este tipo de agencias es el Servicio Nacional de la Mujer (SERNAM), servicio con carácter de ministerio, creado en 1991 para promover la igualdad entre hombres y mujeres ${ }^{12}$.

Estas agencias establecen un vínculo entre el movimiento de mujeres y el gobierno, aunque varían muchísimo en la relación y el espacio que se da a estos movimientos para acceder a la definición de la agenda y a la formulación de políticas públicas, pese a que han sido creadas en gran medida debido a la presión de los movimientos de muje-

\footnotetext{
${ }^{11}$ Para mayor información sobre las tres esferas de poder: toma de decisión, definición de agenda y formación de prioridades, ideas y valores, ver Hay C. Political Analysis. Basingstoke: Palgrave; 2002.

${ }^{12}$ Disponible en http://portal.sernam.cl
} 
res en el mundo $(31,32)^{13}$. Sin perjuicio de estas variaciones, la creación en los países de women's policy agencies ha contribuido tanto al avance en la formulación de políticas que contribuyen a la igualdad de géneros, a dar una señal sobre la importancia de que las mujeres alcancen dicha igualdad, así como a dar un espacio a los movimientos de mujeres en la formulación de políticas públi$\operatorname{cas}(30,33)$.

El acceso de los movimientos de mujeres a la formulación de políticas públicas en asuntos de género es fundamental, debido a que garantiza una mayor representación de los intereses de las mujeres que la hasta ahora escasa participación en puestos parlamentarios y la misma existencia de women's policy agencies cuando no tienen gran capacidad de influenciar políticas públicas. Los movimientos de mujeres son organizaciones transversales, en los que mujeres son sus principales miembros y cuyo objeto es hacer demandas sobre temas de género(34). Estos actores son cruciales para influenciar y definir la agenda pública en torno a los asuntos de género(24,35,36). Esta sería, así concebida, la última forma relevante para las mujeres de lograr influenciar y decidir en políticas sobre asuntos de género. Dado que ingresar a esferas de poder es particularmente difícil para las mujeres y siguen siendo sistemáticamente excluidas de tomar decisiones que directamente les afectan, la participación en movimientos de mujeres y la posibilidad que éstos les dan para presionar por la incorporación de asuntos de género en la agenda pública es fundamental para avanzar en la igualdad de géneros.

\section{Conclusión}

Este artículo busca avanzar en las definiciones de ética global y específicamente debatir sobre sus problemáticas actuales. Particularmente, indaga sobre los vínculos entre concepciones actuales de ética global y temáticas de derechos humanos y género. Con dicho propósito, presenta definiciones de ética y de ética global, así como de derechos humanos. Con ellas se demuestra que estos temas están bastante vinculados y que todo el ámbito de los derechos humanos ha desarrollado

\footnotetext{
${ }^{13}$ En América Latina, además de por la presión de los movimientos de mujeres, las women's policy agencies también han sido creadas por presión internacional(32).
}

e institucionalizado conceptos de ética. También se muestra que desde el punto de los derechos de las mujeres, en específico, hay críticas tanto a concepciones éticas como a los derechos humanos, tal como han sido definidos en un comienzo. Por lo mismo, se ha desarrollado un derecho particular que recoge conceptos y problemáticas específicas que afectan a este sector de la población.

Se señaló que tanto la ética como los derechos humanos están estrechamente vinculados con el concepto de poder, razón por la cual se escogió un derecho específico que se relaciona estrechamente con este concepto: el derecho a la participación política de las mujeres. Se demostró que hay una diferencia bastante importante en la participación política de las mujeres respecto de la de los hombres y se hizo referencia a fórmulas establecidas para una mayor participación política de las mujeres. En dicho contexto, se hizo referencia a la participación parlamentaria y a fórmulas para incrementarla, a las agencias gubernamentales, women's policy agencies, que normalmente favorecen la inclusión de algunos temas de los derechos de las mujeres en la agenda pública, y finalmente se propuso que se puede favorecer ciertos grados de institucionalización del acceso de los movimientos de mujeres a la formulación de la agenda y de políticas públicas.

La mayor participación de las mujeres en la toma de decisiones es un reto que cruza los ámbitos tanto de la ética como de los derechos humanos. Aun queda bastante por lograr, más en el ámbito de la concreción de los derechos que en el de la definición. En dicho proceso se debe tener en cuenta las formas de participación política de las mujeres y los avances logrados, tanto a través de la creación de agencias gubernamentales específicas, que velan por lograr una mayor igualdad entre los sexos, como desde su participación en movimientos de mujeres. Estas características especiales de participación y las maneras de incorporarlas en las formas tradicionales de hacer política son algunos de los aspectos en que la ética puede hacer un aporte a las visiones de los derechos humanos, que hasta ahora no han logrado que estos derechos se transformen en una realidad. 
Referencias

1. Kessler M. Morality. In: Darity Jr, William A. International Encyclopedia of the Social Sciences, $2^{\text {nd }}$ ed. Farmington Hills: Macmillan Reference USA; 2008: 283-286.

2. Drumond JGdeF. Aspectos históricos de la ética. En: Lolas Stepke F, Drumond JGdeF, (eds.) Bioética. Santiago de Chile: Mediterráneo; 2013: 13-20.

3. Coicaud J-M, Warner D. Introduction: Reflections on the Extent and Limits of Contemporary International Ethics. In: Ethics and International Affairs: Extent and Limits. New York: United Nations University Press; 2001: 1-13.

4. Lolas Stepke F, Drumond JGdeF, (eds.) Bioética. Santiago de Chile: Mediterráneo; 2013.

5. Lolas F, Rodríguez E. Bioética: Generalidades y Conceptos Básicos. En: Lolas Stepke F, Drumond JGdeF, (eds.) Bioética. Santiago de Chile: Mediterráneo; 2013: 21-26.

6. Given LM, (ed.) The Sage Encyclopedia of Qualitative Research Methods. United States: Sage; 2008.

7. Twiss SB. Global Ethics and Human Rights: A Reflection. Journal of Religious Ethics 2011; 39(2): 204-222.

8. Lolas Stepke F. Ética global: consideraciones sobre su relevancia en los estudios internacionales. Estudios Internacionales 2013; 174: 7-14.

9. Ackerly B, Attanasi K. Global feminisms: Theory and Ethics for Studying Gendered Injustice. New Political Science 2009; 31(4): 543-555.

10. Donnelly J. Ethics and International Human Rights. In: Coicaud, J-M, Warner D, (eds.) Ethics and International Affairs: Extent and Limits. New York: United Nations University Press; 2001: 128-160.

11. Piechowiak M. What are Human Rights? The Concept of Human Rights and Their Extra-Legal Justification. In: Hanski R, Suksi M, (eds.) An Introduction to the International Protection of Human Rights. A Textbook. $2^{\text {nd }}$, revised ed. Finland: Institute for Human Rights, Abo Akademi University; 1999: 3-14.

12. Freeman M. Human Rights. $2^{\text {nd }}$ Ed. Cambridge: Polity Press; 2011.

13. Smith Rhona KM. Human Rights in International Law. In: Goodhart M, (ed.) Human Rights. Politics and Practice. Oxford: Oxford University Press; 2009: 26-44.

14. Donnelly J. Universal Human Rights. In Theory and Practice. $2^{\text {nd }}$ Ed. United States: Cornell University Press; 2003.

15. Flinterman C, Henderson C. Special Human Right Treaties. In: Hanski R, Suksi M, (eds.) An Introduction to the International Protection of Human Rights. A Textbook. $2^{\text {nd }}$, revised ed. Finland: Institute for Human Rights, Abo Akademi University; 1999: 125-142.

16. Van der Wilt H, Krsticevic V. The OAS System for the Protection of Human Rights. In: Hanski R, Suksi M, Eds. An Introduction to the International Protection of Human Rights. A Textbook. $2^{\text {nd }}$, revised ed. Finland: Institute for Human Rights, Abo Akademi University; 1999: 371-386.

17. Merrills JG. Promotion and Protection of Human Rights within the European Arrangements. In: Hanski R, Suksi M, Eds. An Introduction to the International Protection of Human Rights. A Textbook. $2^{\text {nd }}$, revised ed. Finland: Institute for Human Rights, Abo Akademi University; 1999: 275-286.

18. Finnemore M, Sikkink K. International Norm Dynamics and Political Change. International Organization 1998; 52(4): 887-917.

19. Kent AE. States Monitoring States: The United States, Australia, and China's Human Rights, 1990-2001. Human Rights Quarterly 2001; 23(3): 583-624.

20. Risse Th, Ropp SC, Sikkink K, (eds.) The Power of Human Rights. International Norms and Domestic Change. Cambridge: Cambridge University Press; 1999.

21. Fraser AS. Becoming Human: The Origins and Development of Women's Human Rights Human Rights Quarterly 1999; 21: 853-906.

22. Taylor V. Gender and Social Movements. Gender processes in women's self-help movements. Gender and Society 1999; 13(1): 8-33.

23. Blofield MH, Haas L. Defining a Democracy: Reforming the Laws on Women's Rights in Chile, 1990-2002. Latin American Politics and Society 2005; 47(3): 35-68.

24. Hay C. Political Analysis. Basingstoke: Palgrave; 2002.

25. Ríos Tobar M. Cuotas de género. Democracia y representación. Santiago de Chile: IDEA, FLACSO; 2005.

26. Marx J, Borner J, Caminotti M. Cuotas de género y acceso femenino al parlamento: los casos de Argentina y Brasil en perspectiva comparada. Politica 2006; 46: 61-81.

27. Schwindt-Bayer LA. The Incumbency Disadvantage and Women's Election to Legislative Office. Electoral Studies 2005; 24: 227-244. 
Participación política de las mujeres - Tatiana Rein Venegas

28. Phillips A. Quotas for Women. In: Krook, ML, Childs S, (eds.) Women, Gender, and Politics. A Reader. Oxford: Oxford University Press; 2010.

29. McBride Stetson D, Mazur A, (eds.) Comparative State Feminism. London: Sage Publications; 1995.

30. McBride DE, Mazur AG. The Politics of State Feminism. Innovation in Comparative Research. Philadelphia: Temple University Press; 2010.

31. Molyneux M, Razavi S. Beijing Plus Ten: An Ambivalent Record on Gender Justice. Development and Change 2005; 36(6): 983-1010.

32. Franceschet S, Krook ML. State Feminism and Gender Quotas in the 'North' and 'South': Comparative Lessons from Western Europe and Latin America. Paper prepared for presentation at the Annual Meeting of the International Studies Association San Diego, CA, March 22-25 2006.

33. Blofield M. Women's Choices in Comparative Perspective: Abortion Policies in Late-Developing Catholic Countries. Comparative Politics 2008; 40(4): 399-419.

34. Beckwith K. Mapping Strategic Engagements. International Journal of Politics 2007; 9(3): 312-338.

35. Thompson KB. Women's Rights are Human Rights. In: Sanjeev K, Riker JV, Sikkink K, (eds.) Restructuring World Politics. Transnational Social Movements, Networks, and Norms. Minneapolis and London: University of Minnesota Press; 2002: 96-122.

36. Clark AM, Friedman EJ, Hochstetler K. The Sovereign Limits of Global Civil Society: A Comparison of NGO Participation in UN World Conferences on the Environment, Human Rights, and Women. World Politics 1998; 51(1): 1-35.

Recibido: 26 de septiembre de 2013

Aceptado: 3 de octubre de 2013 